\title{
Wenn Schmerz zur Katastrophe wird
}

\author{
PAIN CATASTROPHIZING SCALE Ob Patienten ihre Schmerzen überbewerten und \\ somit zur Chronifizierung neigen, misst die Pain Catastrophizing Scale zuverlässig und \\ präzise. Therapeuten können sie einsetzen, um maßgeschneiderte Therapien gegen \\ das Katastrophisieren zu planen.
}

E in entscheidender Faktor dafür, ob Schmerzen chronisch werden, ist, ob Patienten dessen Relevanz realistisch einschätzen. Neigen sie dazu, ihn deutlich überzubewerten, also zu katastrophisieren, steigt die Wahrscheinlichkeit einer Chronifizierung [1]. Außerdem kann sich bei diesen Patienten die erfolgreiche Anwendung von Schmerzbewältigungsstrategien um mindestens zwölf Monaten verzögern [12]. Umgekehrt: Bewerten Patienten ihre Beschwerden realistisch, sind die funktionellen Resultate einer Therapie meist besser.

Entsprechend ist es für eine effiziente Therapie wichtig, dass Therapeuten einschätzen können, ob ein Patient katastrophisiert. Dies lässt sich mit der deutschen Version der Pain Catastrophizing Scale (PCS) schnell, einfach und verlässlich herausfinden ( $\odot$ Literaturangabe). Zudem ist die PCS kostenlos [10].

PCS eignet sich bei vielen Erkrankungen > Kanadische Schmerzpsychologen haben die PCS in den 1990er-Jahren für Patienten mit muskuloskeletalen Schmerzen entwickelt [2]. Mittlerweile belegen verschiedene systematische Übersichtsarbeiten, dass die PCS bei vielen unterschiedlichen Krankheitsbildern zuverlässig eingesetzt werden kann: bei unspezifischen Rückenschmerzen [3], nach lumbalen Bandscheibenoperationen [4], nach vorderer Kreuzbandplastik [5], nach akutem HWS-Distorsionstrauma [6], nach Knie- und Hüftendoprothesen [7], aber auch beim chronischen Erschöpfungssyndrom [8] und bei chronischen postoperativen Schmerzen [9].

Die PCS besteht aus 13 Aussagen ( $\odot$ Tab.). Für jede Aussage hat der Patient fünf Antwortmöglichkeiten: von 0 (= trifft überhaupt nicht zu) bis 4 (=trifft immer zu). Der Therapeut addiert die der Antwort entsprechenden Punkte. Der beste Summenscore der PCS ist der minimale mit 0 Punkten (= kein Katastrophisieren), der schlechteste der maximale mit 52 Punkten (= maximales Katastrophisieren). In der Literatur ist kein Punktestand definiert, ab welchem das Ergebnis als kritisch angesehen wird.

Daher gilt: Je höher der Score, desto wahrscheinlicher ist es, dass der Patient katastrophisiert. Patienten brauchen im Durchschnitt fünf Minuten, um den Fragebogen auszufüllen. Die Auswertung durch den Therapeuten von Hand dauert etwa zwei Minuten.

Ich mache mir ständig Sorgen, ob die Schmerzen wohl jemals wieder aufhören werden.

\section{Ich denke ständig daran, wie sehr es schmerzt.}

Ich mache mir Sorgen, dass die Schmerzen auf etwas Schlimmes hindeuten.

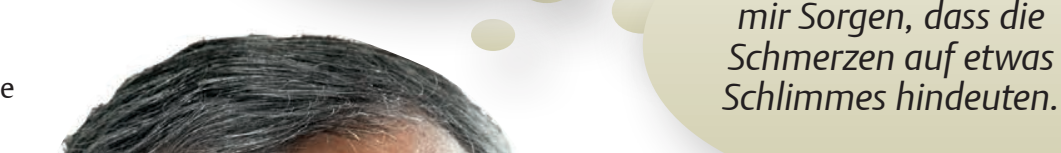




\begin{tabular}{|c|c|c|c|c|c|c|}
\hline & $\begin{array}{l}\text { Wenn ich Schmerzen habe, beschäftigen } \\
\text { mich folgende Gedanken: }\end{array}$ & $\begin{array}{l}\text { Trifft über- } \\
\text { haupt } \\
\text { nicht zu }\end{array}$ & $\begin{array}{l}\text { Trifft eher } \\
\text { nicht zu }\end{array}$ & Teils, teils & Trifft eher zu & $\begin{array}{c}\text { Trifft } \\
\text { immer zu }\end{array}$ \\
\hline 1. & $\begin{array}{l}\text { Ich mache mir ständig Sorgen, ob die Schmerzen } \\
\text { wohl jemals wieder aufhören werden. }\end{array}$ & 0 & 1 & 2 & 3 & 4 \\
\hline 5. & Ich habe das Gefühl, ich halte es nicht mehr aus. & 0 & 1 & 2 & 3 & 4 \\
\hline 11. & $\begin{array}{l}\text { Ich denke ständig daran, wie sehr ich mir ein Ende } \\
\text { der Schmerzen herbeiwünsche. }\end{array}$ & 0 & 1 & 2 & 3 & 4 \\
\hline
\end{tabular}

Tab. Auszug aus der Pain Catastrophizing Scale, deutsche Version [10]

Deutsche Version reliabel und valide > 2008 entwickelten die Schweizer Forscher Anne F. Mannion, Kathrin Meyer und Heike Sprott die deutsche Version des Fragebogens [10]. Sie übersetzten das Original gemäß international anerkannten Leitlinien ins Deutsche und passten die Aussagen an die kulturellen Gegebenheiten im deutschsprachigen Raum an [11].

Die wissenschaftlichen Gütekriterien des PCS sind zuverlässig und valide: Für die Reliabilität konnten Mannion, Meyer und Sprott bei 100 Patienten mit chronischen Rückenschmerzen sehr gute Werte nachweisen [10]. Für die Test-Retest-Reliabilität des gesamten PCS maßen sie einen Intraklassen-Koeffizienten von 0,80 (optimal ist der Wert „1,00“). Um die Konstruktvalidität des PCS zu testen, befragten sie 111 Patienten mit chronischen spezifischen oder unspezifischen Rückenschmerzen. Es zeigte sich, dass die Konstruktvalidität, berechnet mit dem Rangkorrelationskoeffizienten nach Spearman, mit den Werten 0,26 bis 0,61 akzeptabel ist. Optimal wäre der Wert „1,00“.

Mein Patient katastrophisiert. Und jetzt? > Diverse Studien belegen, dass ein erhöhter PCS-Score für Physiotherapeuten sehr relevant ist: Die Patienten haben unter Umständen eine höhere Schmerzintensität, einen erhöhten Medikamentenkonsum, häufiger Depressionen sowie mehr Angst vor Bewegung und Wiederverletzung. Ihr Angst-Vermeidungsverhalten ist stark ausgeprägt, sie sind dekonditioniert, in ihren Alltagsfunktionen eingeschränkt

\section{ZU GEWINNEN}

\section{TENS-EMS-Gerät}

Schmerzlindernde Wirkung hat das 4-Kanal TENS-EMS Kombi Gerät von der Firma axion (www.tens-reizstrom.de). Wir verlosen bis zum 10. April 2014 ein Gerät. Klicken Sie unter www.thieme.de/physiopraxis > „Gewinnspiel“ auf das Stichwort „TENS“.

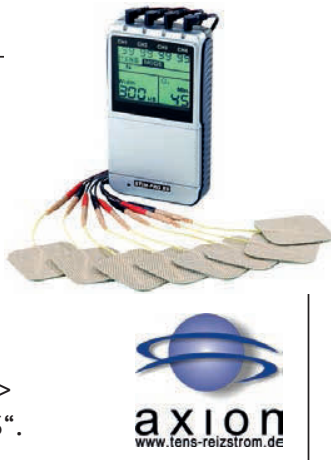

und haben möglicherweise eine erhöhte Sympathikus-Aktivität. Außerdem belegte die Forschungsgruppe von Michael Sullivan, dass bei Patienten nach HWS-Distorsionstrauma mit niedrigem PCS-Score die Chance, zur Arbeit zurückzukehren, fast drei Mal höher war als bei Patienten mit hohem PCS-Score [12].

Die gleiche Forschergruppe zeigte, dass ein Behandlungsprogramm mit Patientenedukation, gemeinsamer Planung von Alltagsaktivitäten und aktiver Bewegungstherapie das Ausmaß des Katastrophisierens (gemessen mit dem PCS) verringert [13]. Folglich sollte der Fokus in der Therapie auf den Umgang und die Bewältigung der Erkrankung ausgerichtet sein. Zudem können Therapeut und Patient gemeinsam dessen körperliche Belastungen unter die Lupe nehmen. Der Therapeut schult den Patienten darin, Überbelastungen zu vermeiden und achtsam mit seinen körperlichen Ressourcen im Alltag umzugehen. Durch dosierte Bewegungstherapie, etwa nach den Prinzipien der graduierten Exposition, bekommt der Patient ein positives Körpergefühl vermittelt, was den Selbstwert stärkt, das Katastrophisieren reduziert und die Lebensqualität steigert [14]. Martin L. Verra und Balz Winteler

\section{$\Rightarrow$ Das Literaturverzeichnis sowie die komplette „Pain Catastrophi- zing Scale“ (deutsche Version) finden Sie unter www.thieme- connect.de/ejournals/physiopraxis > "Ausgabe 3/14“.}

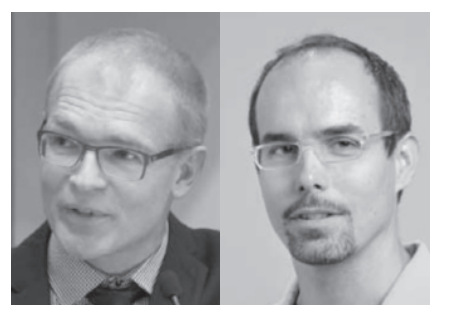

Martin L. Verra, PhD, PT, ist Direktor des Instituts für Physiotherapie am Inselspital, Universitätsspital Bern, Schweiz (www.physio.insel.ch), und Vizepräsident der Stiftung Physiotherapie Wissenschaften (www.physiotherapie-wissenschaften.ch). Balz Winteler, MSc, PT, ist Schwerpunktleiter Muskuloskelettal des Instituts für Physiotherapie am Inselspital, Universitätsspital Bern, Schweiz. Daneben ist er Präsident des Schweizerischen Verbands Orthopädischer Manipulativer Physiotherapie (www.svomp.ch).

Beide Autoren haben langjährige Erfahrung in der Behandlung von Patienten mit chronischen muskuloskeletalen Beschwerden. 